\title{
In the Wake of the "Implant Files": Unheard Ideas to Make Device-Based Therapies safer and always more Innovative
}

\author{
Egron Sandrine* \\ Co-founder and CEO at InnYTe, Health Tech Hub. Former Research Associate at Dept. of Cardiovascular Engineering, Institute of Applied Medical \\ Engineering Helmholtz Institute, RWTH Aachen University / University Hospital Aachen, Germany.
}

*Corresponding author: Egron Sandrine, Co-founder and CEO at InnYTe, Health Tech Hub. Former Research Associate at Dept. of Cardiovascular Engineering, Institute of Applied Medical Engineering Helmholtz Institute, RWTH Aachen University | University Hospital Aachen, Germany.

To Cite This Article: Egron Sandrine. In the Wake of the "Implant Files": Unheard Ideas to Make Device-Based Therapies safer and always more Innovative. Am J Biomed Sci \& Res. 2019 - 4(1). AJBSR.MS.ID.000750. DOI: 10.34297/AJBSR.2019.04.000750

Received: July 02, 2019 | Published: July 15, 2019

\section{Introduction}

The invention of implantable devices has revolutionized how doctors treat their patients. The case of faulty aortic valve replacement illustrates the spectacular progress which has been achieved. The first prosthetic aortic valve was implanted in 1952 by Dr. Hufnagel. The valve was made of a rigid cylindrical bulb chamber containing an acrylic ball. The prosthetic valve, which was placed in the descending aorta during a high-risk surgical procedure, improved the conditions of hundreds of patients in the fifties, though it offered poor hemodynamics and patients could hear the valve's prosthetic ball bouncing around in the chamber [1].

Today's transcatheter aortic valve replacement (TAVR) prostheses use biological tissue to form three valve leaflets, thereby imitating the native valve and optimizing treatment outcome. The TAVR valves are commonly implanted through a minimally invasive femoral artery access in less than an hour. In the last decade, this revolutionary technology has been used to treat around 400,000 patients worldwide [2]

The medical device industry continues to invest in developing better solutions for patients thus transforming and improving treatments. Earlier this year, the US Food and Drug Administration (FDA) showed a promising 9\% annual growth in the number of novel device approvals during the period 2009-2018 [3].

There is however a cloud on the horizon: last year's release of the "Implant Files"-resulting from an on-going global investigation led by the International Consortium of Investigative journalists (ICIJ) [4] cast a shadow on the regulatory framework for medical devices and brought into light the serious safety issues posed by several implantable devices. Public confidence in medical products was shaken by multiple scandals in the pharmaceutical industry. The medical device industry, together with healthcare authorities and the medical community, have ways to avoid the dangerous pitfalls unveiled along decades of pharmaceutical research and development which brought major medical progress. This year, health authorities across Western and Asian countries pledged strong reforms on medical device approval and post-market surveillance regulatory frameworks. For example, the FDA has started modernizing the controversial $510 \mathrm{k}$ procedure [5]. But is the reinforcement of regulations the only answer? The FDA is committing to more transparency and released databases last June containing millions of reports on devices which may have malfunctioned or caused a death or serious injury [6].

Although the databases present limitations, these new tools can greatly help clinicians, researchers, and manufacturers discuss and prevent devices risks. Still, the on-going efforts to improve patient protection may fall short. Here are two initiatives which could be fostered by health authorities to make device-based therapies safer. First, reinforce clinicians' abilities to debate potential risks posed by the newest technologies. Second, publish comparative data on the devices which can be used for the same medical indication as early as possible.

\section{Empowering the Clinicians in the Device Risks Debate}

At the start of my career as an engineer and researcher in the cardiovascular field, I discovered the knowledge gap that exists between the engineers who design devices and the clinicians who pick devices for their patients and implant them. The engineers are often unaware of clinical parameters which matter to the treatment outcome, while the clinicians are undertrained on the technical concepts which explain the device functioning and properties. I recall discussions with cardiologists and heart surgeons to whom I described the blood flow dynamics across one 
device or the material properties of another device which could affect the implantation procedure outcome. While these concepts seemed obvious to my engineer-trained mind, they were not to most clinicians. By supplementing their medical experience with device-related technical knowledge, clinicians could play a greater role in anticipating potential risks posed by a device and ask more informed questions to companies bringing new devices to the hospital.

Currently, companies provide high-quality training on how to use their proprietary devices. One session explaining the technical concepts governing the device's behavior could be added to these trainings and made available online. Also, modernizing the technical courses included in medical university curriculums, to match the practical questions faced by clinicians in their current device use would help equip the soon-to-become clinicians, and reinforce their future role in the development of innovative and safe devices.

\section{Choosing the Right Medical Device}

One practical question, which clinicians face for all implantation procedures, is which device to pick for the patient. For example, a clinician who decides to replace a damaged aortic valve percutaneously can pick among no less than seven competitive TAVR devices [7]. Choosing a device would not be an issue if all devices presented the same guarantees of safety and efficacy for the patient. But unlike generic drugs which are made of the exact same active ingredient as the original drug and yield the same therapeutic effect, competitive devices differ in design, materials, and manufacturing process, and they do not present the same mechanical properties. All these characteristics potentially impact the short and long-term treatment outcome [8].

Several registries and clinical trials are bringing valuable comparative data on available devices. Clinicians can, for example, deduce from this acquired experience that one device functions better to replace a native bicuspid valve, or another device is safer to use in a highly calcified anatomy. Unfortunately, the registries' and trials' information are incomplete, preventing a full understanding of the device panel. Besides, this information becomes available only several years after the devices hit the market.

Implantable devices present an advantage over molecules: they can be looked at, touched, and bench tested. Valuable information could be gained early by running comparative experimental tests for all available devices. These tests could be organized by independent actors or industry consortiums. Showing and discussing the results with the medical community in open sessions, while continuously challenging the testing methods as technologies evolve, could benefit all the actors in the medical device field.

Product development cycles for devices are shorter than for drugs. The medical device industry moves fast from an old device generation to a newer one, while multiple companies bring in competitive devices for the same medical indications. It is essential that the devices which stay on the market are the ones which guarantee the best treatment outcomes for the patients. All clinicians can help the health authorities and the medical device companies achieve this goal. By empowering clinicians with robust technical knowledge and data, the trend towards device-based therapy breakthroughs will accelerate. In this dawn of an era of digital and connected medicine, the need for transparency and shared knowledge among the technical and clinical communities has become even greater.

\section{References}

1. P Rajashekar (2015) Development of mechanical heart valves-an inspiring tale. J Pract Cardiovasc Sci 1(3): 289-293.

2. Panagiota Kourkoveli, Konstantinos Spargias, George Hahalis (2018) TAVR in 2017-What we know? What to expect? J Geriatr Cardiol. 15(1): 55-60.

3. (2019) Statement from FDA Commissioner Scott Gottlieb MD and Jeff Shuren MD, Director of the Center for Devices and Radiological Health, on a record year for device innovation.

4. The ICIJ (International Consortium of Investigative Journalists), a USbased nonprofit organization, encompasses 249 investigative reporters from more than 90 countries and territories.

5. https://www.fda.gov/news-events/press-announcements/statementfda-commissioner-scott-gottlieb-md-and-jeff-shuren-md-directorcenter-devices-and-1

6. (2019) Medical Device Reporting (MDR): How to Report Medical Device Problems.

7. Denise Todaro, Andrea Picci, Marco Barbanti (2017) Technical characteristics and evidence to date for FDA- and CE Mark-approved valves. Cardiac Interventions Today 11(2).

8. L Anderson (2018) Generic Drug FAQs. 